\title{
ELECTRONIC AND THERMOELECTRIC PROPERTIES IN LI-BASED HALF- HEUSLER COMPOUNDS: A FIRST PRINCIPLE STUDY
}

\author{
Y. Benazouzi ${ }^{1}$, H. Rozale ${ }^{1 *}$, M. A. Boukli Hacene ${ }^{1}$, M. Khethir ${ }^{1}$, A. Chahed ${ }^{1}$, and D. \\ Lucache $^{2}$ \\ ${ }^{I}$ Condensed Matter and sustainable development Laboratory (LMCDD), University of Sidi Bel-Abbes, Sidi Bel- \\ Abbes 22000, Algeria. \\ ${ }^{2}$ Faculty of Electrical Engineering "Gh.Asachi” Technical University Iasi, Romania \\ *Corresponding author: Email: hrozale@yahoo.fr
}

\begin{tabular}{|l|l|}
\hline \multicolumn{1}{|c|}{ Article Info } & \multicolumn{1}{c|}{ Abstract } \\
\hline Received: 18.11 .2019 & \multicolumn{1}{|c|}{ In this paper, we performed a first principle study for new half-Heusler } \\
Accepted: 31.12 .2019 & $\begin{array}{l}\text { LiSrX(X= N, P, and As) working with WIEN2k code in the frame work of the } \\
\text { density functional theory, and the Boltzmann theory. We estimated the exchange- } \\
\text { correlation potential by the generalized gradient approximation (GGA). }\end{array}$ \\
$\begin{array}{l}\text { Keywords: } \\
\text { Li-based half-Heusler } \\
\text { compounds, DFT, } \\
\text { Thermoelectric } \\
\text { properties }\end{array}$ & $\begin{array}{l}\text { Energetically, the three compounds show a high stability in structure type2, we } \\
\text { notice that the lattice constant increased while bulk modulus decreased in replacing } \\
\text { the ions of size increasing. Based on our calculations, LiSrN, LiSrP, and LiSrAs } \\
\text { compounds are mechanically stable, and show semiconductor nature with indirect } \\
\text { band gaps of 1.21, 1.75 for LiSrN and LiSrAs, and direct band gap of 1.94 eV for } \\
\text { LiSrP. The thermoelectric properties are calculated for LiSrX (X=N, P, and As) } \\
\text { and they found a high power factor for the p-type doping concentration. }\end{array}$ \\
\hline
\end{tabular}

\section{Introduction}

The exhaustion of fossil fuels, the economic development and environmental have push the scientists to search another alternative for the new sources of renewable energy; this search has been mainly motivated by the introduction of new materials thermoelectric for energy conversion in small-scale power generation and refrigeration devices, not only for industrial sectors, but for the human body as well. In fact, good thermoelectric materials devices depend mostly on the type of materials used and their properties such as electrical conductivity, thermal stability, thermal conductivity, and the Seebeck coefficient.

The ternary compounds containing the $\mathrm{Li}$ atom in the form of semi-Heusler materials have recently attracted much attention, because of their potential use in the production of thermoelectricity, spintronics, opto-electronics, etc. [1, 2, 3, 4, 5, 6, and 7]. This type of 
Heusler compounds attracted much attention as promising semiconductors for electronic devices of high efficiency power. The semi-Heusler lithium-based compounds are a wide band gap (direct and indirect) semiconductors and a high PF power factor, they have interesting properties such as their adjustable band gap, which can be modified from 0 to 4 $\mathrm{eV}$ by the difference of electronegativity of its constituents [3]. Because of these particular properties they have received wide attention, especially in the field of solar cells and thermoelectric devices.

Very recently Syed Hatim Shah et al. [8] studied the optoelectronic and thermoelectric properties of $\mathrm{LiBZ}$ semiconductors $(\mathrm{B}=\mathrm{Al}, \mathrm{In}, \mathrm{Ga}$ and $\mathrm{Z}=\mathrm{Si}, \mathrm{Ge}, \mathrm{Sn})$, they found that the Seebeck coefficient of this compound has a behavior of type $n$ and type $p$, the maximum value of the Seebeck coefficient decreases by increasing the temperature, and a high thermal conductivity but moderate as a function of temperature.

Recently, Rashid Ahmed et al. [9] give a condensed overview on the diverse capabilities of the Heusler family of Type $\mathrm{XMgN}$, they noticed that the investigated materials revealed high values of Seebeck coefficient at $300 \mathrm{~K}$, and it was observed to be decreased with the temperature increase; the impact of temperature on electrical conductivity has been relatively low.

Joachim Barth et al. [10] studied the Seebeck coefficient and the resistivity of LiAlSi and LiAlGe compounds in the temperature range from $2 \mathrm{~K}$ to $650 \mathrm{~K}$. They found a maximum value of the Seebeck coefficient at a temperature of $400 \mathrm{~K}$ for LiAlSi compound, and small thermal conductivity for LiAlGe. Finally they concluded that the LiAlSi is more promising of the two compounds.

Deepika Shrivastava et al. [11] have studied the structural, electronic, phonon and thermoelectric properties of $\mathrm{KScX}(\mathrm{X}=\mathrm{Sn}$ and $\mathrm{Pb})$ and $\mathrm{KYX}(\mathrm{X}=\mathrm{Si}$ and $\mathrm{Ge})$ half-Heusler compounds. They found that all half-Heusler compounds have high Seebeck coefficient and high-Power Factor for p-type. The electrical conductivity increases linearly with temperature which confirms the semiconducting nature of these compounds.

To investigate and understand the properties of these Heusler compounds, it is necessary to correctly assign the atomic positions in the crystal lattice. In general, the halfHeusler phases crystallize in a non-centro symmetric structure corresponding to the space $F \overline{4} 3 m$, No. 216. The structure type is $\mathrm{MgAgAs}$ or $\mathrm{C}_{b}$. The half-Heusler structure is a ternary ordered variant of the $\mathrm{CaF}_{2}$ crystal structure. In the half-Heusler structure, within the lattice, the atoms on Wyckoff positions $4 \mathrm{a}(0,0,0)$ and $4 \mathrm{~b}(1 / 2,1 / 2,1 / 2)$ form the ionic NaCl-type substructure, while the atoms on $4 \mathrm{a}$ and $4 \mathrm{c}(1 / 4,1 / 4,1 / 4)$ build the covalent $\mathrm{ZnS}$-type one [4]. 
In Table 1, the positions occupied by the three atoms and the vacancy are given according to the notations defined by Wyckoff [12].

To the best of our knowledge, $\operatorname{LiSrX}(\mathrm{X}=\mathrm{N}, \mathrm{P}$, and As) at the present time, the halfHeusler compounds which are the subject of this study have not been studied. For this reason we expect our theoretical results to serve as a source for experimental testing.

In this letter we present the study of structural, electronic, elastic and thermoelectric properties of $\mathrm{LiSrX}(\mathrm{X}=\mathrm{N}, \mathrm{P}$, and $\mathrm{AS})$ compounds have been investigated using fullpotential linearized augmented plane wave method.

Table 1. Inequivalent site occupancies within the $C 1_{b}$-type structure for $\operatorname{LiSr} X(X=N, P$ and As).

\begin{tabular}{llll}
\hline & $\mathrm{A}$ & $\mathrm{B}$ & $\mathrm{C}$ \\
\hline $\begin{array}{l}\text { Structure } \\
\text { Type } 1\end{array}$ & $4 \mathrm{c}(1 / 4,1 / 4,1 / 4)$ & $4 \mathrm{~b}(1 / 2,1 / 2,1 / 2)$ & $4 \mathrm{a}(0,0,0)$ \\
Type 2 & $4 \mathrm{~b}(1 / 2,1 / 2,1 / 2)$ & $4 \mathrm{a}(0,0,0)$ & $4 \mathrm{c}(1 / 4,1 / 4,1 / 4)$ \\
Type 3 & $4 \mathrm{a}(0,0,0)$ & $4 \mathrm{c}(1 / 4,1 / 4,1 / 4)$ & $4 \mathrm{~b}(1 / 2,1 / 2,1 / 2)$ \\
Zinc blende & - & $4 \mathrm{a}(0,0,0)$ & $4 \mathrm{c}(1 / 4,1 / 4,1 / 4)$ \\
\hline
\end{tabular}

\section{Calculations Methods}

In this study, the calculations were done using FP-LAPW computational scheme [13, $14]$ as implemented in the WIEN2K code [15]. For exchange-correlation (XC) potential the generalized gradient approximation (Perdew-Burke-Ernzerhof (PBE-GGA) are used [16]. The muffin-tin sphere radii were chosen to be 2.22 a.u. for Li, 2.46 a.u for N, P, and As, and 2.25 for Sr, For the Brillion zone (BZ) integration, the tetrahedron method [15] with a 104 special $\mathrm{k}$ points in the irreducible edge (3000 k -points in the full BZ) was used to construct the charge density in each self-consistency step. For thermoelectric properties the semiclassical Boltzmann theory as embedded in the BoltzTrap code [17] was employed.

\section{Results and discussions}

In order to predict the equilibrium lattice constants of Li-based half-Heusler LiSrX $(\mathrm{X}=\mathrm{N}, \mathrm{P}$, and As) alloys, the structural properties of compounds were calculated by optimizing the total energy with respect to the volume for three possible atomic arrangements 
(Type 1, 2, 3); these parameters are calculated and fitted according to the Murnaghan's equation of state [18].

Table2 presents the calculated structural properties (equilibrium lattice constant $a_{0}$, the bulk modulus $B$, and its first derivative $\left.B^{\prime}\right)$ of half-Heusler compounds $\operatorname{LiSrX}(\mathrm{X}=\mathrm{N}, \mathrm{P}$, and As) compared with other results $[2,5,6,7]$.

Table2. Calculated structural and elastic properties of half-Heusler compounds $\operatorname{LiSr} X(X=N$, $P$, and As) compared with other results.

\begin{tabular}{lccc}
\hline \hline & LiSrN & LiSrP & LiSrAs \\
\hline $\boldsymbol{a}\left(\boldsymbol{A}^{\circ}\right)$ & 5.89 & 6.81 & 6.98 \\
& $5.54[2]$ & $6.49[2]$ & $6.84[6]$ \\
& $5.01[7]$ & $6.02[5]$ & $6.67[2]$ \\
& $5.02[5]$ & & $6.21[5]$ \\
$\boldsymbol{B}(\boldsymbol{G P a})^{\boldsymbol{M}}$ & 57.32 & 35.64 & 32.11 \\
$\boldsymbol{B}^{\prime}$ & 4.1 & 3.98 & 4 \\
$\boldsymbol{E}_{\boldsymbol{g}}$ & 1.21 & 2.09 & 1.74 \\
& & & $1.93[6]$ \\
$\boldsymbol{C}_{\boldsymbol{1 1}}(\boldsymbol{G P a})$ & 136.4 & 69.23 & 64 \\
$\boldsymbol{C}_{\boldsymbol{1 2}}(\boldsymbol{G P a})$ & 17.19 & 19.06 & 16.12 \\
$\boldsymbol{C}_{44}(\mathbf{G P a})$ & 21.85 & 23.19 & 24.57 \\
$\boldsymbol{B}(\boldsymbol{G P a})$ & 56.95 & 35.78 & 32.08 \\
$\boldsymbol{S}_{\boldsymbol{v}}$ & 36.96 & 23.95 & 24.31 \\
$\boldsymbol{Y}$ & 91.17 & 58.74 & 58.23 \\
$\boldsymbol{v}$ & 0.23 & 0.22 & 0.19 \\
$\boldsymbol{A}$ & 0.54 & 0.92 & 1.02 \\
\hline \hline
\end{tabular}

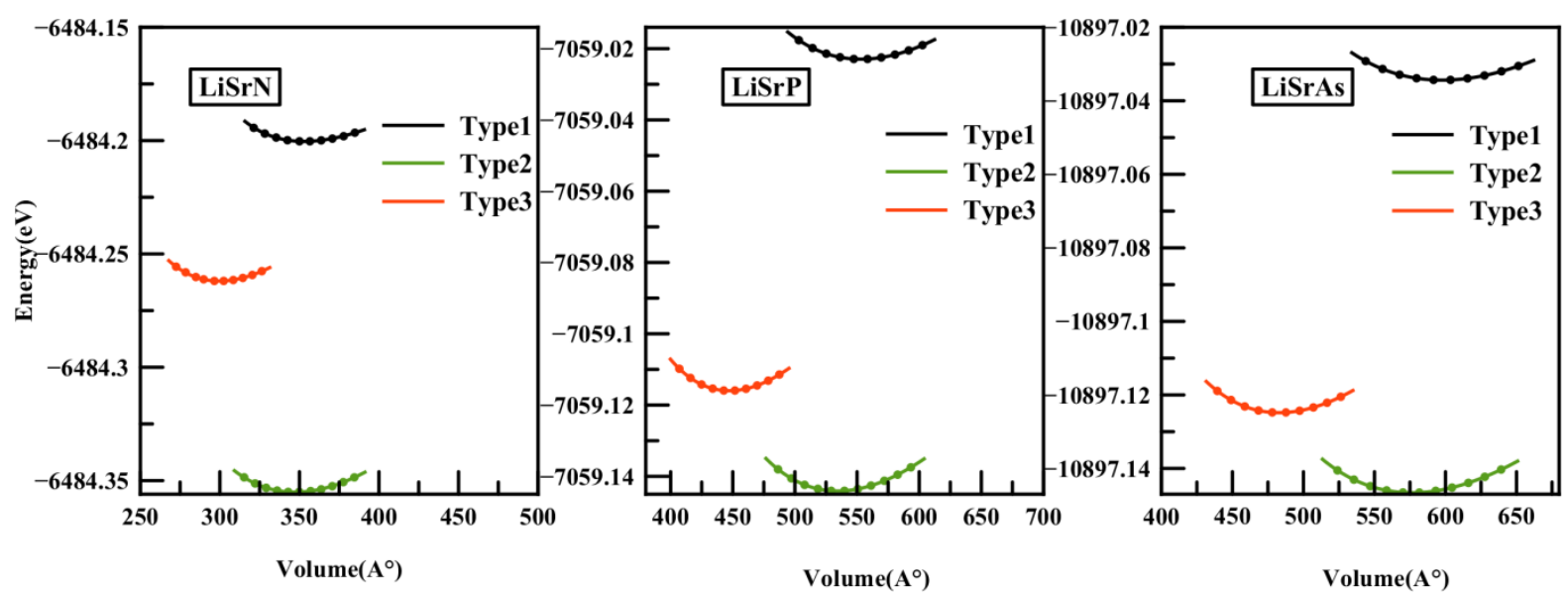

Fig. 1. Volume optimization for cubic (MgAgAs-type) phase for (a) LiSrN, (b) LiSrP, (c) LiSrAs half-Heusler compounds. 
To examine the mechanical stability of the compounds studied, the elastic properties are calculated for a cubic structure using IRelast package [19] as implemented in the WIEN2K code [15], there are three independent elastic constants $C_{11} ; C_{12}$ and $C_{44}$. To calculate these constants, three independent types of stress are applied: uniform, tetragonal and rhombohedral. For solid materials with cubic symmetry the mechanical stability is determined by the following criteria: $\mathrm{C}_{11}>0 ; \mathrm{C}_{11}>\mathrm{C}_{22} ; \mathrm{C}_{11}+2 \mathrm{C}_{12}>0$ and $\mathrm{C}_{44}>0$.

Calculated elastic constants of $\operatorname{LiSrX}(\mathrm{X}=\mathrm{N}, \mathrm{P}$, and As) half-Heusler compounds are listed in Table2., the calculated values satisfy the criteria motioned above, confirms that the compounds are mechanically stable, and they noticed that the $\mathrm{C}_{11}$ constant is considerably higher than the $\mathrm{C}_{12}$ and $\mathrm{C}_{44}$ constants, indicating that the materials have higher resistance to compression in the $\mathrm{x}$-direction.

The calculate elastic constants $\mathrm{C}_{11} ; \mathrm{C}_{12}$ and $\mathrm{C}_{44}$ permit us to obtain other elastic constants $[20,21,22,23,24,25]$ such as the Voigt shear modulus $\left(S_{v}\right)$, Voigt bulk modulus $(B), B / S_{v}$ ratio, Voigt Young's modulus $(Y)$, Voigt Poisson's ratio $(V)$ and anisotropic factor (A) are presented in Table 2. The Bulk $(B)$ or shear modulus $(S)$ measures the hardness of materials [26].

The bulk modulus is 56.95, 35.78, and 32.08 GPa for LiSrN, LiSrP, and LiSrAs, respectively. To examine the ductility and brittleness of the material, the $B / S$ ratio was calculated, if this ratio is greater than 1.75 the material behaves in a ductile nature, otherwise it behaves brittle [26]. In the present calculations, the $B / S_{v}$ ratio of $\operatorname{LiSrX}(\mathrm{X}=\mathrm{N}, \mathrm{P}, \mathrm{As})$ halfHeusler are $1.54,1.49$ and 1.31 , respectively, depending on the $B / S v$ ratio results, the three compounds have brittle nature. In order to examine the stiffness of the materials, the Young's modulus (Y) has been calculated, a high value of this module implies a strong covalent bonds which implies the rigidity of the materials. Our results indicate that stiffness decreases in the following order: $\mathrm{LiSrN}>\mathrm{LiSrP}>\mathrm{LiSrAs}$. This decreasing order may be due to the decrease of covalent bond strength between $\mathrm{Sr}$ atom and $\mathrm{X}$ atoms. Also we calculated the Poisson's ratio $s(v)$ which measures the stability of the material. When the Poisson's ratio $(v)$ is less than $1 / 3$, the material behaves brittle; otherwise it is ductile [27]. One can see that the Poisson's ratio (v) for ours half-Heusler alloys $\operatorname{LiSrX}(\mathrm{X}=\mathrm{N}, \mathrm{P}$, and As) are 0.23, 0.22, and 0.19 respectively, indicated that the three compound are brittle. The elastic anisotropic (A) is a another fundamental parameter for confirming the stability, in general way if (A) equal to one the material is isotropic, otherwise, the material is anisotrope. The calculated elastic anisotropic 
factor for $\mathrm{LiSrN}$ and $\mathrm{LiSrP}$ compounds is different than 1, which indicates that these compounds are not elastically isotropic; on the other hand it's elastically isotropic for LiSrAs.

To study and understand the electronic state of our compounds, we calculated the band structures of LiSrX (X=N, P, As) half-Heusler alloys along the high symmetry directions in the Brillouin zone. Figure 2(a, b, and c) shows the calculated band structure using (GGAPBE) method. From the figure, it is observed that the valence band maximum for $\mathrm{LiSrN}$ and LiSrAs compounds lies at the X-point, whereas the conduction band minimum lies at the $\Gamma$ point, indicate that $\operatorname{LiSrX}(\mathrm{N}, \mathrm{As})$ materials are semiconductor with an indirect band gap, but for LiSrP the valence band maximum and the conduction band minimum lies at the X-point, indicates that the LiSrP compound has a direct band gap. The obtained band gaps of GGAPBE methods and other previous data are listed in Table 2; the calculated band gaps are 1.21, 2.09, and 1.74 for LiSrN, LiSrP, and LiSrAs compounds respectively.

To fully understand the electronic band structure of the half-Heusler alloys in this study, their total and partial density of states are also presented. The plots are shown in Figure 3(a, b, and c). The overall profile of TDOS and PDOS are similar for all three compounds. As shown in the figures, in the considered energy range the lithium atom does not participate in the hybridization, while the maximum of the valence band from $-1.70 \mathrm{eV}$ to $0 \mathrm{eV}$ is represented by the hybridization between $\mathrm{N}-2 p$ and $\mathrm{Sr}-5 s$ states, $\mathrm{P}-3 p$ and $\mathrm{Sr}-5 s$ states, $\mathrm{P}-3 p$ and Sr-5s states, As- $4 p$ and Sr-5 $\underline{s}$ states. The lower partitions from $-11 \mathrm{eV}$ to $-10 \mathrm{eV}$ in $\mathrm{LiSrN}$, from $-8.1 \mathrm{eV}$ to $-7.2 \mathrm{eV}$ in $\mathrm{LiSrP}$ and $-6.69 \mathrm{eV}$ to $-7.95 \mathrm{eV}$ in LiSrAs are mainly due to $\mathrm{N}-2 s$, $\mathrm{P}-3 s$ and As- $4 s$ states, respectively. In all cases, the conduction band starting from the band gap is dominated mainly by the $\mathrm{Sr}-5 s$ state.

The thermoelectric properties of materials depends on the electronic band structure of LiSrX (X=N, P, and As) half-Heusler alloys and especially at the level of the maximum of the valence band (MBV).

Figure (4(a), 5(a) and 6(a)) shows the variation of the Seebeck coefficient, also called the thermoelectric power, of $\operatorname{LiSrX}(\mathrm{X}=\mathrm{N}, \mathrm{P}$, and $\mathrm{As})$ alloys pounds as a function of the chemical potential for different temperatures $300 \mathrm{k}, 600 \mathrm{~K}$ and $900 \mathrm{~K}$.

The positive (negative) values of this parameter shows the $\mathrm{p}(\mathrm{n})$-type with holes(electrons) as main carriers. The figure shows that in the range of chemical potential considered, the Seebeck coefficient has significant values in both negative and positive regions, which indicates that both types of carriers may be implicate, and it is clear that the value of the thermoelectric power has large values for low values of the chemical potential. The maximum values of Seebeck coefficient are listed in Table .3. 


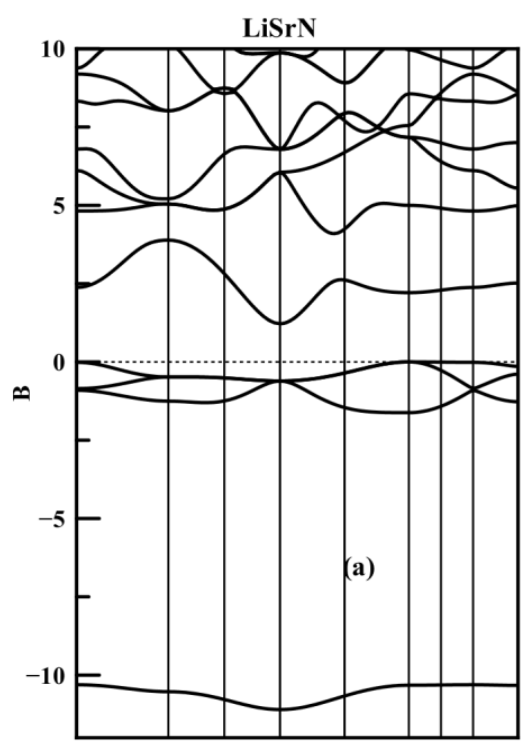

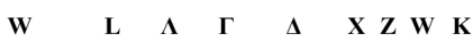

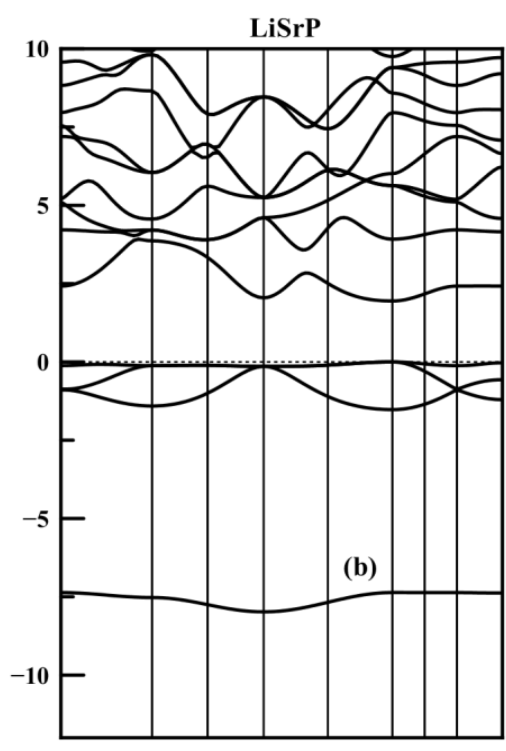

$\begin{array}{llllllllll}\mathbf{W} & \mathbf{L} & \boldsymbol{\Lambda} & \boldsymbol{\Gamma} & \boldsymbol{\Delta} & \mathbf{X} & \mathbf{Z} & \mathbf{W} & \mathrm{K}\end{array}$

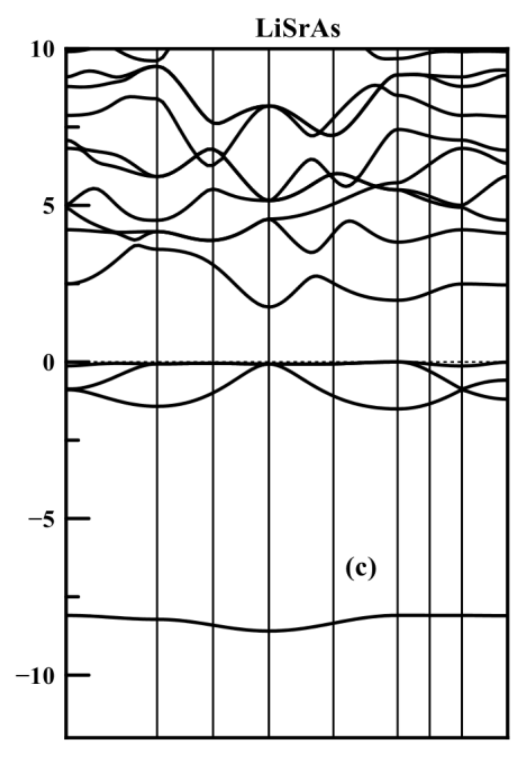

$\begin{array}{llllllllll}\mathbf{W} & & \mathbf{L} & \boldsymbol{\Lambda} & \boldsymbol{\Gamma} & \boldsymbol{A} & \mathbf{X} & \mathbf{Z} & \mathbf{W} & \mathbf{K}\end{array}$

Fig. 2. Electronic band structure in cubic (MgAgAs-type) phase for (a) LiSrN, (b) LiSrP, (c) LiSrAs half-

Heusler compounds.

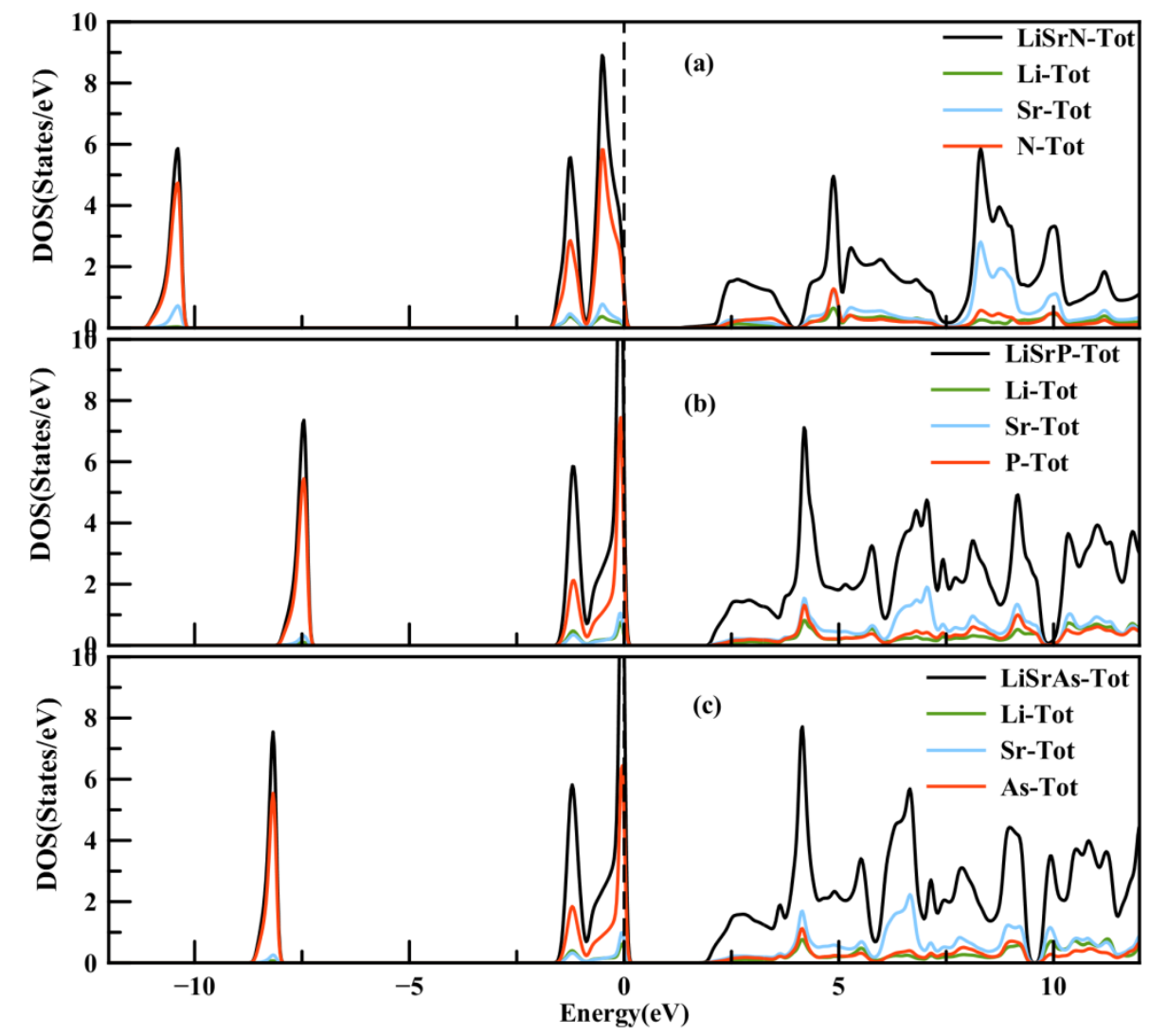

Fig. 3. The total and tolal atoms DOS in cubic (MgAgAs-type) phase for (a) LiSrN, (b) LiSrP, (c) LiSrAs halfHeusler compounds 
From the Table 3 we can see when we increase the temperature from $300 \mathrm{~K}$ to $900 \mathrm{~K}$, the maximum value of Seebeck coefficient decreases greatly as much as 50.19\%(35.91\%), 75.32\%(72.88\%), and 66.19\%(58.08\%) for p-type (n-type) in case of LiSrN, LiSrP and LiSrAs, respectively. From Temperature $300 \mathrm{~K}$ to $900 \mathrm{k}$ we see that the Seebeck coefficient decreases dramatically for the regions (type n, type p) in the case of the compound LiSrN compare with the two compounds $\operatorname{LiSrX}(\mathrm{X}=\mathrm{P}, \mathrm{As})$.

Table 3. Maximum values of Seebeck coefficient $S(\mu \mathrm{V} / \mathrm{K})$; Electric conductivity $\sigma\left(10^{19} / \mathrm{Qms}\right)$; Electric thermal conductivity $\boldsymbol{\kappa}\left(10^{14} \mathrm{w} / \mathrm{mk}^{2} \mathrm{~s}\right)$ and power factor per relaxation time $\left(* 10^{10}\right)$ of $\operatorname{LiSrX}(X=N, P$, and As $)$ half-Heusler compound

\begin{tabular}{lcccccccc}
\hline \multicolumn{7}{c}{ p-Type } & \multicolumn{7}{c}{ n-Type } \\
& S & $\boldsymbol{\sigma}$ & $\boldsymbol{\kappa}$ & $\mathbf{P F}$ & $\mathbf{S}$ & $\boldsymbol{\sigma}$ & $\boldsymbol{\kappa}$ & $\mathbf{P F}$ \\
\hline LiSrN & & & & & & & & \\
300K & 1534 & 15.35 & 1.08 & 49 & -1534 & 34.83 & 2.51 & 14.18 \\
$600 \mathrm{~K}$ & 1112 & 14.86 & 1.88 & 85 & -876 & 34.41 & 4.78 & 42.2 \\
$900 \mathrm{~K}$ & 770 & 14.06 & 2.17 & 106 & -551 & 33.15 & 6.56 & 71.4 \\
& & & & & & & & \\
\hline LiSrP & & & & & & & & \\
$300 \mathrm{~K}$ & 1475 & 15.26 & 1.05 & 28 & -1475 & 31.33 & 2.27 & 13.99 \\
$600 \mathrm{~K}$ & 1567 & 14.76 & 1.86 & 66 & -1567 & 30.79 & 4.38 & 41 \\
$900 \mathrm{~K}$ & 1111 & 13.98 & 2.29 & 94 & -1075 & 30.27 & 6.15 & 74 \\
\hline LiSrAs & & & & & & & & \\
$300 \mathrm{~K}$ & 1553 & 14.44 & 1.11 & 44 & -1553 & 29.91 & 2.11 & 10.8 \\
$600 \mathrm{~K}$ & 1488 & 14.95 & 1.96 & 73 & -1457 & 29.30 & 4.08 & 34 \\
$900 \mathrm{~K}$ & 1028 & 14.21 & 2.45 & 97 & -902 & 28.77 & 5.74 & 64 \\
\hline
\end{tabular}

The movement of electrons from high-temperature regions to the low-temperature regions causes an electric current. High electrical conductivity value implies good materials for thermoelectric applications, the plots of electrical conductivity of $\operatorname{LiSrX}(\mathrm{X}=\mathrm{N}, \mathrm{P}$ and $\mathrm{As})$ compounds as a function of chemical potential at temperatures $300 \mathrm{~K}, 600 \mathrm{~K}$, and $900 \mathrm{~K}$ are displayed in Figure (4(b), 5(b) and 6(b)). The electrical conductivity in positive chemical potential region is higher than that in the negative region. This result shows that the electron doping in these $\operatorname{LiSrX}(\mathrm{X}=\mathrm{N}, \mathrm{P}, \mathrm{As})$ alloys will be more advantageous for their thermoelectric performance than hole doping, at $300 \mathrm{~K}$ the maximum value of electrical conductivity is $15.4\left(10^{19} / \Omega \mathrm{ms}\right)$ at $-0.028 \mathrm{eV}, 15.26\left(10^{19} / \Omega \mathrm{ms}\right)$ at $-0.035 \mathrm{eV}$, and $14.2\left(10^{19} / \Omega \mathrm{ms}\right)$ at $-0.028 \mathrm{eV}$ for LiSrN, LiSrP and LiSrAs, respectively. It is also perceived 
that raising the temperature slightly decreases the electrical conductivity of these half-Heusler alloys, which can be explained by the increasing carrier concentration plus collisions and scattering phenomenon at high temperatures.
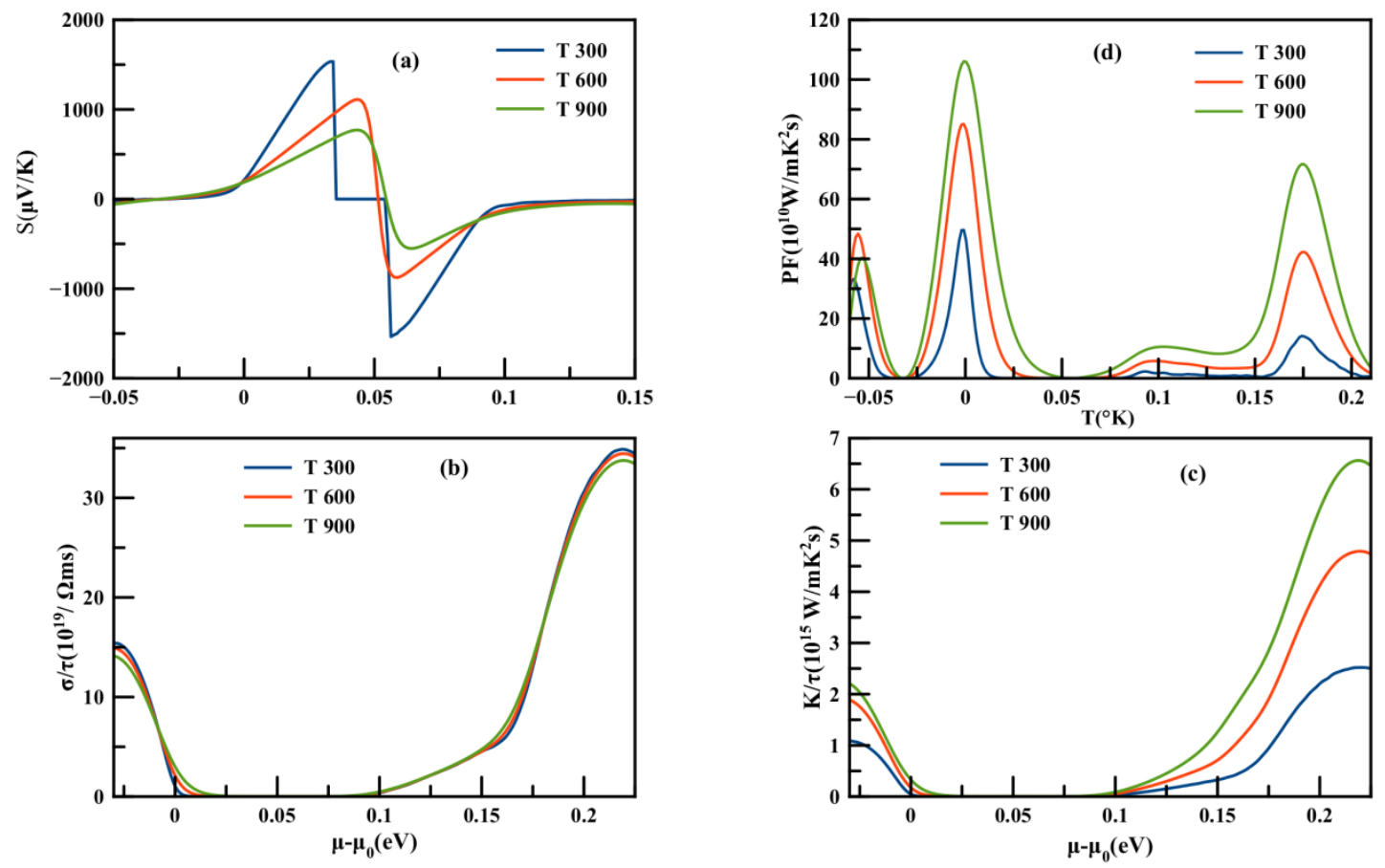

Fig. 4. Thermoelectric properties, (a) Seebeck coefficient, (b) Electric conductivity, (c) Thermal conductivity, (d) and Power factor par relaxation time of LiSrN half-Heusler compound
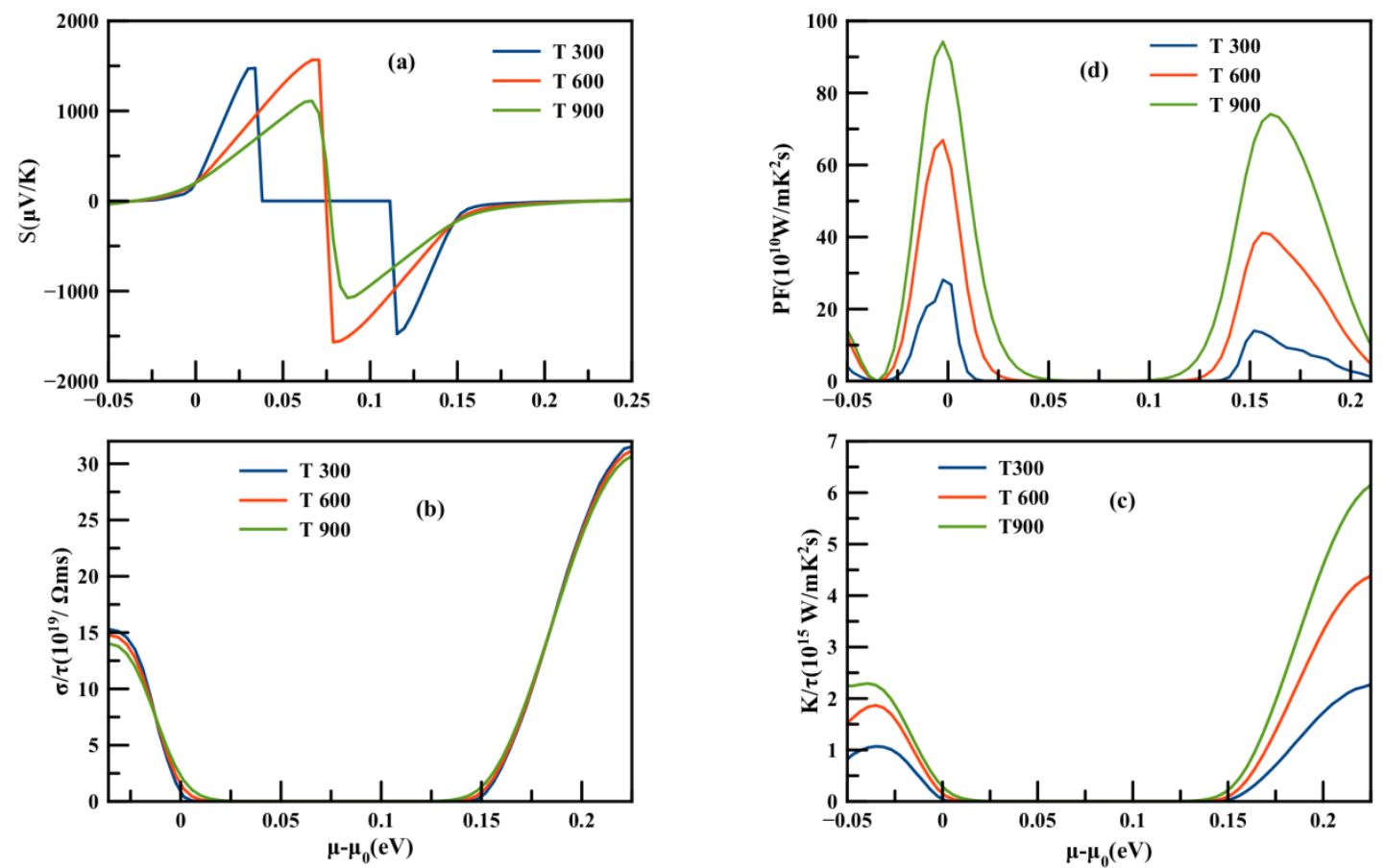

Fig. 5. Thermoelectric properties, (a) Seebeck coefficient, (b) Electric conductivity, (c) Thermal conductivity, (d) and Power factor par relaxation time of LiSrP half-Heusler compound 
In this work, only the electronic contribution to thermal conductivity of $\operatorname{LiSrX}(X=\mathrm{N}, \mathrm{P}$ and As) half-Heusler alloys is considered. In Figure (4(c), 5(c) and 6(c)), the variation of electronic thermal conductivity as a function of chemical potential at temperatures $300 \mathrm{~K}$, $600 \mathrm{~K}$, and $900 \mathrm{~K}$ is illustrated. It is notice that the plots of both electrical and electronic thermal conductivity have very similar profile. These results agree with the WiedemannFranz law, which states the proportional relation between them as follows: $k=\sigma L T$ [28]. However, unlike electrical conductivity, electron thermal conductivity increases dramatically with increasing the temperature from $300 \mathrm{~K}$ to $900 \mathrm{~K}$. At $300 \mathrm{~K}$, the maximum value of this parameter for p-type is $1.09\left(10^{15} \mathrm{~W} / \mathrm{mK}^{2} \mathrm{~s}\right), 1.86\left(10^{15} \mathrm{~W} / \mathrm{mK}^{2} \mathrm{~s}\right)$ and $2.17\left(10^{15} \mathrm{~W} / \mathrm{mK}^{2} \mathrm{~s}\right)$ in the case of LiSrN, LiSrP and LiSrAs, respectively. While for the $n$ type, it continuous is increasing even at chemical potentials higher than $0.21 \mathrm{eV}$.
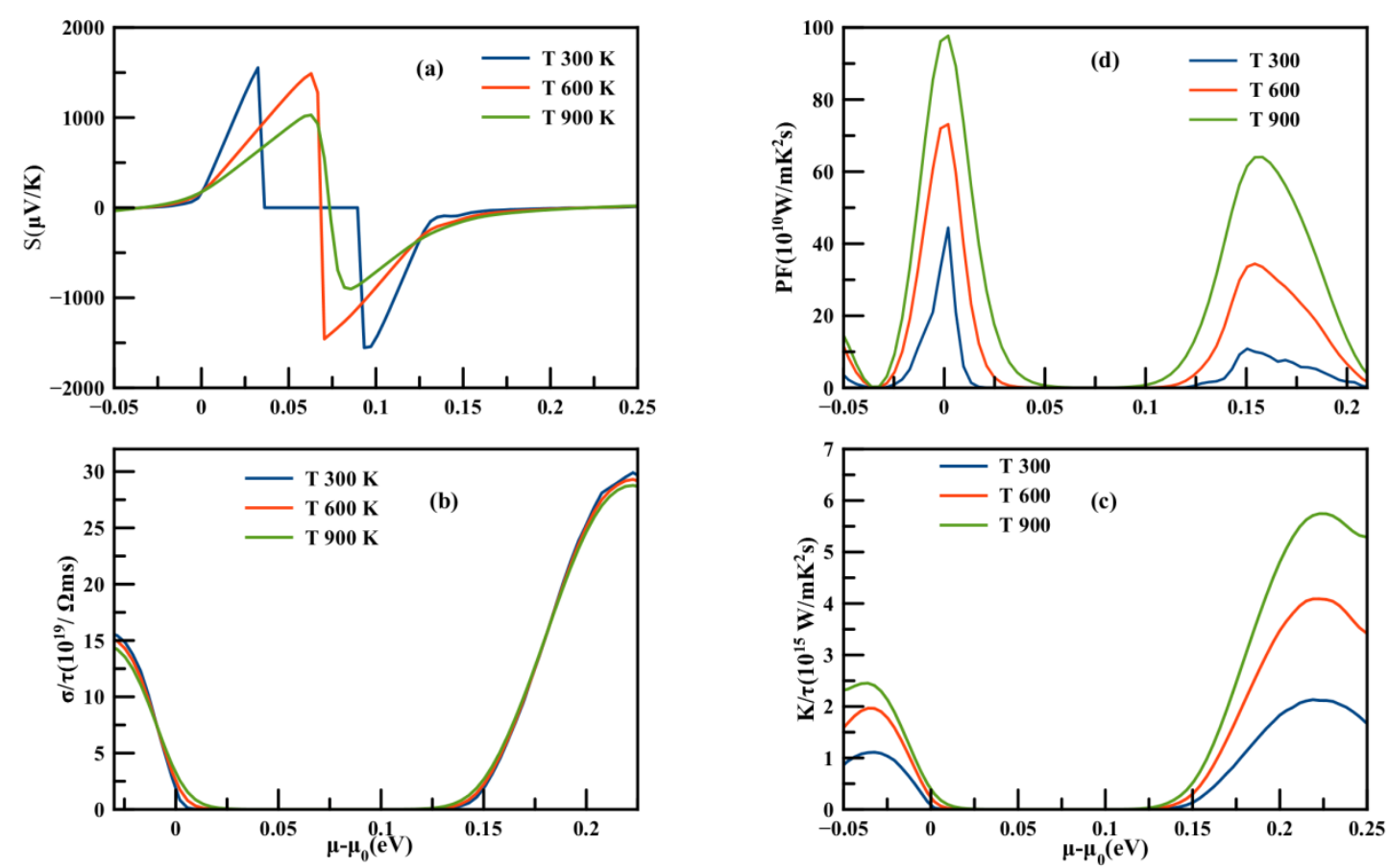

Fig. 6. Thermoelectric properties, (a) Seebeck coefficient, (b) Electric conductivity, (c) Thermal conductivity, (d) and Power factor par relaxation time of LiSrAs half-Heusler compound

The PF power factor is a significant amount to examine the efficiency of the thermoelectric material. It can be determined by the information of Seebeck coefficient and electrical conductivity $\left(\mathrm{PF}=\mathrm{S}^{2} \sigma / \tau\right)$. Figure $(4(\mathrm{~d}), 5(\mathrm{~d})$ and $6(\mathrm{~d}))$, shows the graphs of calculated power factor with respect to relaxation time for $\operatorname{LiSrX}(X=N, P$ and $A s)$. For the studied materials and at $300 \mathrm{~K}$ the curves show that the minimum of the power factor is required. Though, we notice that if the temperature increases the power factor values increase 
and become a maximum at $900 \mathrm{~K}$. The calculated maximum power factors versus chemical potential have been listed in Table 3. At $900 \mathrm{~K}$ temperature, the maximum power factor for $\mathrm{p}$ type are $106 \times 10^{10} \mathrm{~W} / \mathrm{mk}^{2} \mathrm{~s}(-0.0008 \mathrm{eV}), 94 \times 10^{10} \mathrm{~W} / \mathrm{mk}^{2} \mathrm{~s}(-0.0025 \mathrm{eV})$ and $97 \times 10^{10} \mathrm{~W} / \mathrm{mk}^{2} \mathrm{~s}(-$ $0.0019 \mathrm{eV})$; while for n-type the values are $71.4 \times 10^{10} \mathrm{~W} / \mathrm{mk}^{2} \mathrm{~s}(0.17 \mathrm{eV}), 74 \times 10^{10} \mathrm{~W} / \mathrm{mk}^{2} \mathrm{~s}$ $(0.16 \mathrm{eV})$ and $64.0 \times 10^{10} \mathrm{~W} / \mathrm{mk}^{2} \mathrm{~s}(0.15 \mathrm{eV})$ of $\mathrm{LiSrN}, \mathrm{LiSrP}$ and LiSrAs, respectively. At room temperature, the $\mathrm{LiSrN}$ has the maximum power factor compared to those of $\mathrm{LiSrP}$ and LiSrAs compounds, this is due to their large value of DOS.

\section{Conclusions}

To summarize our work: we have explored the structural, elastic, electronic, and thermoelectric properties of LiSrN, LiSrP, and LiSrAs half-Heusler alloys using the first principles calculations based on FP-LAPW method and Boltzmann transport theory. It has been found that energetically the most stable state is type 2 for the three compounds; we remarked that the lattice constant is elevated while bulk modulus decreased in replacing the ions of size increasing. Our results indicate that all three compounds are mechanically stable, and they are more resistant to the unidirectional strain than to the shear strain, our results given indicate that the stiffness decreases in the following order: $\mathrm{LiSrN}>\mathrm{LiSrP}>\mathrm{LiSrAs}$, this decreasing order may be due to the decrease of covalent bond strength between $\mathrm{Sr}$ atom and $\mathrm{X}$ atoms. For the thermoelectric properties the Seebeck coefficient has significant values in both positive and negative regions, which suggests that both carrier types can be involved. The results show that the power factor values for a $p$-type region of the investigated materials are higher than $n$-type region.

\section{References}

[1] Hem Chandra Kandpal, Claudia Felser and Ram Seshadri, J. Phys. D: Appl. Phys.39 (2006) 776-785

[2] S. Kacimi, H. Mehnane, A. Zaoui, Journal of Alloys and Compounds 587 (2014)451-458

[3] F. Casper, T. Graf, S. Chadov, B. Balkeand, and C. Felser, Semicond. Sci.Technol.27 (2012) 063001

[4] L. Damewood, B. Busemeyer, M. Shaughnessy, C. Y. Fong, L. H. Yang, and C. Felser, Phys. Rev. B 91(2015) 064409

[5] Thomas Gruhn, Physical Review B82 (2010)125210

[6]Anindya Roy, Joseph W. Bennett, Karin M. Rabe, and David Vanderbilt, Physical Review Letters 109, (2012)037602 
[7] R. Umamaheswari, M. Yogeswari, G. Kalpana, Journal of Magnetism and Magnetic Materials 350(2014)167

[8] Shah, S. H., Khan, S. H., Laref, A., and Murtaza, G. Journal of Solid State Chemistry, 258(2018) 8008

[9] Ahmed, R., Masuri, N. S., UlHaq, B., Shaari, A., Alfaifi, S., Butt, F. K., and all. Materials and Design 136(2017)196

[10] Barth, J., Fecher, G. H., Schwind, M., Beleanu, A., Felser, C., Shkabko, A., and all. Journal of Electronic Materials 39 (2010)1856

[11] Deepika Shrivastava, Sankar P. Sanyal, Journal of Alloys and Compounds 784 (2019)319

[12] R. W. G. Wyckoff, Crystal Structures, 2nd ed., Vol. 1(John Wiley \& Sons, (1963)

[13] Madsen G.K.H., Blaha P., Schwarz K., Sjöstedt E., Nordström L., Phys. Rev. B, 64 (2001) 195134

[14] Schwarz K., Blaha P., Madsen G. K. H., Comput. Phys. Commun., 147 (2002) 71

[15] Blaha P., Schwarz K., Madsen G.K.H., Kvasnicka D., Luitz J., Wien2k: An Augmented Plane Wave + Local Orbitals Program for Calculating Crystal Properties, Karlheinz Schwarz/Techn. Universität Wien, Austria, (2001)

[16] Perdew J.P., Burke K., Ernzerhof M., Phys. Rev. Lett., 77 (1996) 3865

[17] G.K.H. Madsen, D.J. Singh, Comput. Phys. Commun. 175 (1) (2006) 67

[18] F. Birch, J. Appl. Phys. 9 (1938) 279

[19] M. Jamal, S.J. Asadabadi, I. Ahmad, H.R. Aliabad, Comput. Mater. Sci. 95 (2014) 592

[20] S. Wang, H. Ye, Phys. Status Solidi 240 (2003) 45

[21] B. Mayer, H. Anton, E. Bott, M. Methfessel, J. Sticht, J. Harris, P. Schmidt, Intermetallic 11 (2003) 23

[22] A. Maachou, H. Aboura, B. Amrani, R. Khenata, S.B. Omran, D. Varshney,Comput. Mater. Sci. 50 (2011)3123

[23] M. Hachemaoui, R. Khenata, A. Bouhemadou, A.H. Reshak, D. Rached, F. Semari, Curr. Opin. Solid State Mater. Sci. 13 (2009)105

[24] A. Bouhemadou, R. Khenata, Phys. Lett. A. 362(2007)476

[25] D. M. Hoat, J.F. R. Silva, A.M. Blas, Mater. Res. Express 5 (6) (2018)066549

[26] D.M. Teter, MRS Bull. 23 (1998) 22.

[27] S. F. Pugh, the London, Edinburgh and Dublin Philosophical Magazine and, J. Sci. 45 (1954) 823.

[28] Bejan, A. \& Allan, A. D. Heat Transfer Handbook (Wiley, New York, 2003), p. 1338 